Part of Journal of Research of the National Bureau of Standards, Volume 13, September 1934

\title{
DRIFT OF MAGNETIC PERMEABILITY AT LOW INDUCTIONS AFTER DEMAGNETIZATION
}

\author{
By Raymond L. Sanford
}

ABSTRACT

The magnetic permeability of ferromagnetic materials at low values of induction depends upon the time which elapses between demagnetization and testing. The change may be of the order of 10 or 12 percent. In order to obtain consistent and reproducible results in testing at low inductions, a period of from 18 to 24 hours should elapse after demagnetization before the test is made.

\section{CONTENTS}

I. Introduction

II. Testing methods

III. Observations and results

IV. Summary

\section{INTRODUCTION}

In the standard methods of test for the magnetic properties of iron and steel adopted in $1933^{1}$ by the American Society for Testing Materials, two alternative methods are given for the determination of permeability and core loss at very low values of induction. It is obvious that unnecessary disputes are likely to arise unless the two methods give substantially the same results for identical specimens. In order to determine whether or not this requirement is fulfilled, tests on a number of samples of various materials were made by the two methods. The results of these tests appeared to indicate that the two methods did not give satisfactory agreement. The differences however, were not consistent either in magnitude or sign. A search for the reason for the discrepancies finally led to the conclusion that the cause lay in the behavior of the material rather than in the methods themselves. It was found that if consistent and reproducible results are to be obtained, a certain definite procedure must be followed. If this is done, the results obtained by the two methods on the same sample agree within reasonable limits.

\section{TESTING METHODS}

The two methods employ alternating current and are designated as the bridge method and the potentiometer method, respectively. The specimen is of the same form for either method and consists of strips of sheet material $25 \mathrm{~cm}$ long and $3 \mathrm{~cm}$ wide built up in the form of a

1 ASTM Standards, 404(1933). 
square with butt and lap joints at the corners. The strips are inserted in forms upon which there are two uniformly distributed windings of 100 and 1,000 turns, respectively. In the bridge method only one of the coils is used, the choice depending upon the type of material to be tested. In the potentiometer method both coils are used, one as a primary and the other as a secondary.

The bridge is a parallel Owen bridge by which values of selfinductance and apparent resistance of the test winding are determined. The permeability of the specimen is calculated in terms of the measured inductance and the core loss is calculated from the excess of the apparent resistance of the test winding over its ohmic resistance. The maximum induction is proportional to the voltage applied to the bridge and measured with a suitable voltmeter. For tests at 60 cycles per second, a vibration galvanometer is used as a detector.

The potentiometer is of the coordinate type which indicates voltage in terms of two components having a quadrature phase relation. The maximum induction is calculated in terms of the voltage induced in the secondary winding. The phase relations are adjusted so that this voltage is in phase with one of the components of the potentiometer. The two components of the drop across a standard shunt connected in series with the primary winding are then determined. The magnetizing force is calculated in terms of the component in quadrature with the induced voltage and the loss is calculated in terms of the in-phase component taking into consideration the ratio of the number of turns in the two windings. One of the essential conditions for either test is that the applied voltage shall have approximately a sine wave form, with not more than 10 percent of total harmonics present. The range of inductions for which these methods apply is from 10 to 1,000 gausses.

Before making a test by either method, the material is demagnetized with 60-cycle alternating current which is gradually reduced from a value sufficient to bring the material into the third stage of magnetization to a value lower than the lowest to be used in making a measurement. It is not necessary to repeat the demagnetizing process provided that points are taken in the order of increasing values of magnetizing force.

\section{OBSERVATIONS AND RESULTS}

Tests were made in the range of induction from 10 to 100 gausses on several samples of silicon steel and hipernik. In some cases it appeared that the values of permeability for a given value of maximum induction as determined by the potentiometer were higher than those determined by the bridge method. On the other hand, it was sometimes found that the higher results were obtained by the bridge method. Moreover, check tests by either method seldom agreed with the original results obtained by the same method, the differences sometimes amounting to as much as 10 or 12 percent.

A clue as to the source of the difficulty was found in an article by S. P. Boudrine ${ }^{2}$ in which it was stated that the value of permeability obtained depends upon the interval of time which elapses between demagnetization and the test. This phenomenon has also been

\footnotetext{
${ }^{2}$ Boudrine, Trv. de l'Insu. de Met. et Stand. de l' U.R.S.S.,4, 77(1933).
} 
noted by Wild and Perrier ${ }^{3}$ and by Steinhaus. ${ }^{4}$ Its importance with respect to practical magnetic testing has been emphasized by the increasing use of magnetic materials at very low values of induction and by the development of methods of testing materials at low inductions on a routine basis.

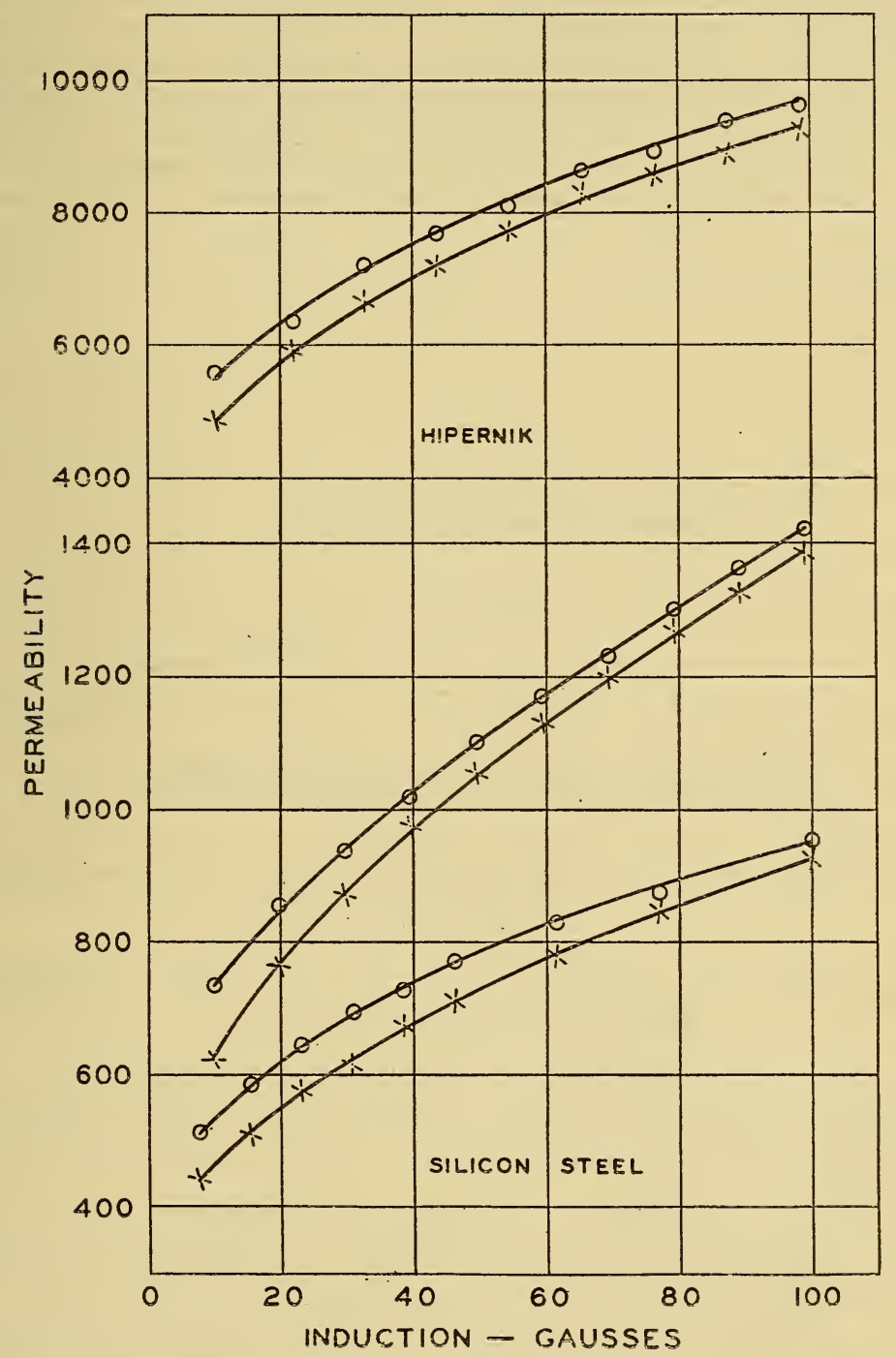

FIGURE 1.-Showing differences in permeability obtained by testing immediately and 18,19 , or 20 hours after demagnetization.

Circles represent points obtained immediately and crosses represent points obtained after the lapse of time.

The results obtained with three typical materials will serve to illustrate the phenomenon. Curves for two types of silicon steel and for a sample of hipernik are shown in figure 1 . The data for the

${ }^{3}$ Wild and Perrier, Arch. d. Scien. Phys. et. Nat., 7,209(1926).

'Stienhaus, Z. f. Tech. Phys. 7,192(1926). 
upper curves were taken immediately after demagnetization and in as short a time as possible. The lower curves were taken 18, 19, and 20 hours, respectively, after the specimens had been demagnetized. Experiments showed that the results did not depend upon the method of demagnetization.

The change in permeability is most rapid immediately after demagnetization and progresses at a continually decreasing rate as time goes on. This is illustrated in the upper curve of figure 2 which shows the relation between permeability and time after demagnetization at a constant value of induction of 50 gausses for silicon steel no. 1. The magnetizing current was off between readings. At the end of 20 hours the permeability had decreased by 10.4 percent.

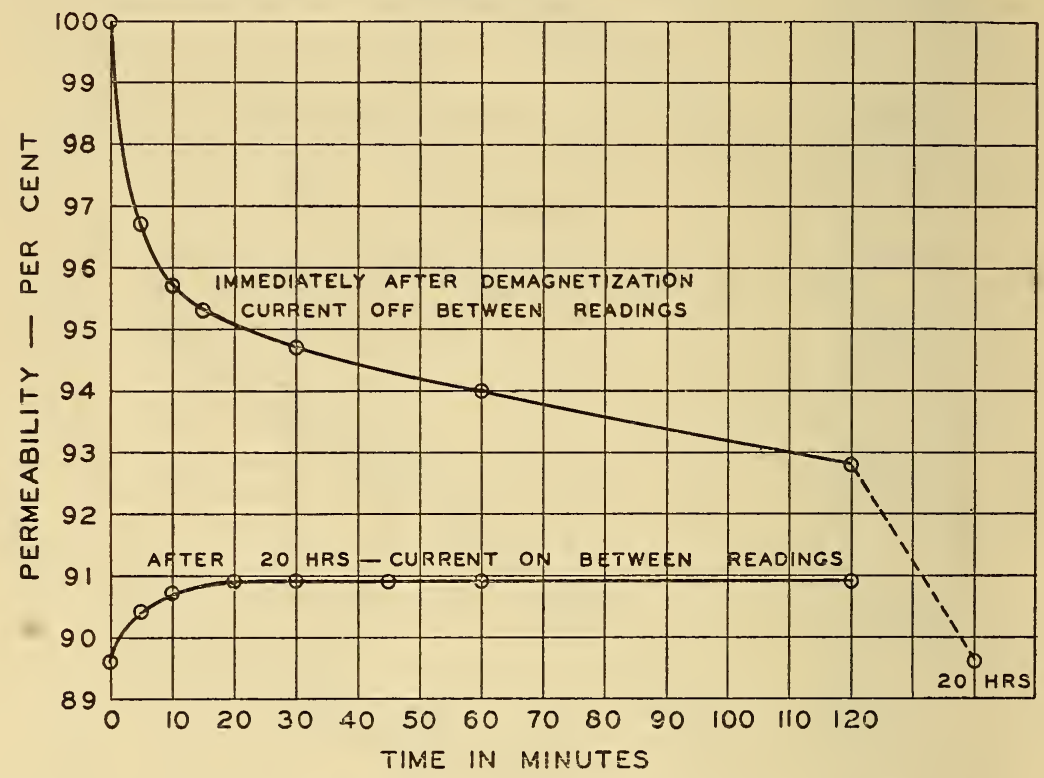

FIGURE 2.-Change of permeability with time elapsed after demagnetization as observed at an induction of 50 gausses.

Half of this decrease took place during the first 30 minutes. If the alternating magnetizing current is kept on continuously, the permeability decreases, but less rapidly than when the current is off between readings.

At the end of a period of 20 hours, measurements were made at intervals over a period of 2 hours with the magnetizing current kept on continuously. The result is shown in the lower curve in the figure. The permeability increased during the first 20 minutes and then remained at a constant value. The total change in this instance, however, was only 1.3 percent.

The change in permeability is not associated primarily with alternating-current magnetization. Measurements were made on the same sample of silicon steel by the direct-current ballistic method. The results obtained at a constant magnetizing force of 0.036 oersteds are shown in figure 3 . In this case the permeability decreased 12.6 
percent in 2 hours. The magnetizing current was on only during the time necessary to make a reading.

Although the primary object of the investigation was to determine how closely the results obtained by the two testing methods on identical samples should be expected to agree and to establish a procedure for obtaining consistent and reproducible results, it may be of interest to consider some of the possible causes of the observed change in permeability. The iron-nickel alloys of which hipernik is an example have a decided tendency to be less stable magnetically than the iron-silicon alloys. It would not have been surprising, therefore, if it had been found difficult to obtain reproducible results with the iron-nickel alloys only. It appears, however, that the phenomenon is just as pronounced in the iron-silicon alloys. It cannot be considered, therefore, to be a characteristic of any particular type of material. Other possible causes which might be considered are mag-

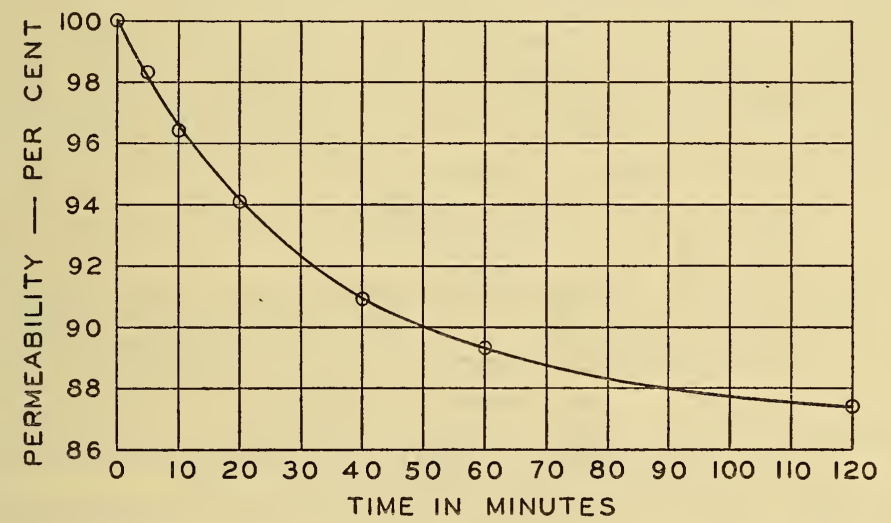

FIGURE 3.-Change of permeability with time elapsed after demagnetization observed by the ballistic method at a magnetizing force of 0.036 oersteds.

netic viscosity, aging, and temperature changes. The effect is entirely different from magnetic viscosity and the materials investigated are of the kinds in which viscosity effects are usually not pronounced. Silicon steel is generally considered to be practically nonaging. One of the samples tested is known to have been in the laboratory for at least 20 years, during which time any aging which might take place should be practically complete. Furthermore, since the change is reversible and can be reproduced at will, the phenomenon should not be ascribed to aging effects. Neither should it be ascribed to ordinary temperature effects. The magnitude of the change is much greater than could be accounted for in terms of the temperature coefficient of magnetic permeability.

It might be thought that the phenomenon is related in some way to the influence of the earth's field, which is from 10 to 20 times as great as the magnetizing forces used in the measurements. However, the drift was observed even when the specimen was so oriented as to bring the earth's field at right angles to that of the magnetizing coils.

Although no theoretical explanation of the phenomenon is offered, the immediate and practical objects of the investigation have been 
attained. Results reproducible within 1 or 2 percent are satisfactory for ordinary commercial testing. This degree of reproducibility can be obtained by waiting from 18 to 24 hours after demagnetization before making a test. By following this procedure the results shown in figure 4 were obtained. These show that the agreement between the two methods both as to permeability and core loss is within the allowable experimental error.

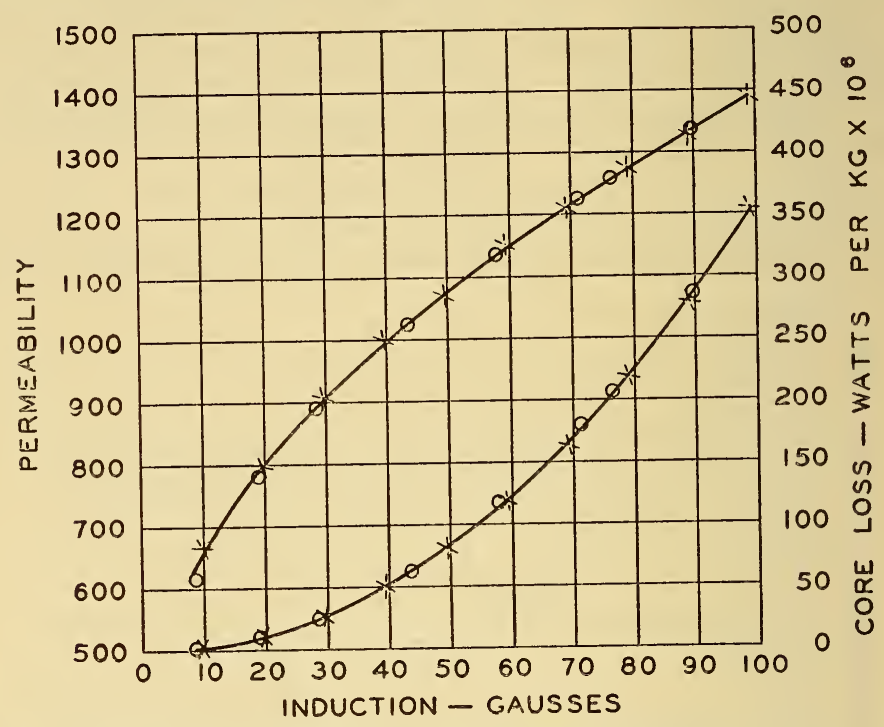

FIgURE 4.-Values of permeability (upper curve) and core loss (lower curve) obtained 24 hours after demagnetization.

Circles represent points obtained by the bridge method. Crosses represent values obtained by the potentiometer method.

\section{SUMMARY}

Since the standard specifications for magnetic testing recently adopted by the American Society for Testing Materials permit the use of either of two alternative methods for tests at very low inductions, it is important that results obtained on the same sample by the two methods shall be in agreement. Tests carried out for the purpose of determining whether or not this requirement is fulfilled showed an apparent lack of agreement which was finally traced to the behavior of the material rather than to any systematic difference between the two methods. It has been found that at very low inductions the magnetic permeability decreases with time after demagnetization. The rate of change is a maximum immediately after demagnetization and decreases as time goes on. The total change may be of the order of 10 to 15 percent. If a sufficient length of time (18 to 24 hours) is allowed to elapse between demagnetization and testing, it is possible to obtain consistent and reproducible values both of permeability and core loss. When this precaution is taken, values obtained on the same specimen by the two methods permitted by the specifications of the American Society for Testing Materials should be in agreement within the allowable experimental error.

Washington, June 5, 1934. 